\title{
Clinical and genetic data of Huntington disease in Moroccan patients
}

\author{
Ahmed Bouhouche ${ }^{1,2}$, Wafaa Regragui ${ }^{1,2}$, Hind Lamghari ${ }^{3}$, Khadija Khaldi ${ }^{4}$, Nazha Birouk ${ }^{5}$, \\ Safaa Lytim², Soufiane Bellamine ${ }^{6}$, Yamna Kriouile 7 , Naima Bouslam², El Hachmia Ait Ben Haddou, ${ }^{1,2}$, \\ Mustapha Alaoui Faris ${ }^{6}$, Ali Benomar ${ }^{1,2}$, Mohamed Yahyaoui ${ }^{1,2}$
}

1. Equipe de Recherche sur les Maladies Neurodégénératives, Faculté de Médecine et de Pharmacie, Université Mohammed V de Rabat, Morocco

2. Service de Neurologie et de Neurogénétique, Hôpital des Spécialités de Rabat, Morocco

3. Centre de diagnostic polyvalent, Salé, Morocco

4. Centre de diagnostic, CHP, Rabat, Morocco

5. Service de Neurophysiologie clinique, Hôpital des Spécialités de Rabat, Morocco

6. Service de Neurologie et de Neuropsychologie, Hôpital des Spécialités de Rabat, Morocco

7. Service de Pédiatrie, Hôpital d'enfant, Rabat, Morocco

\begin{abstract}
Background: Huntington's disease (HD) occurs worldwide with prevalence varying from 0.1 to $10 / 100,000$ depending of the ethnic origin. Since no data is available in the Maghreb population, the aim of this study is to describe clinical and genetic characteristics of Huntington patients of Moroccan origin.

Methods: Clinical and genetics data of 21 consecutive patients recruited from 2009 to 2014 from the outpatient clinic of six medical centers were analyzed. Statistical analysis was performed using descriptive statistics.

Results: Twenty one patients from 17 families were diagnosed positive for the IT15 gene CAG expansion. Clinical symptoms were predominantly motor (19/21). Twelve patients had psychiatric and behavioral disorders, and 11 patients had cognitive disorders essentially of memory impairment. Analysis of genetic results showed that 5 patients had reduced penetrant (RP) alleles and 16 had fully penetrant (FP) alleles. The mean CAG repeat length in patients with RP alleles was 38.4 \pm 0.54 , and $45.37 \pm 8.30$ in FP alleles. The age of onset and the size of the CAG repeat length showed significant inverse correlation ( $\mathrm{p}<0.001, \mathrm{r}=-0.754)$.

Conclusion: Clinical and genetic data of Moroccan patients are similar to those of Caucasian populations previously reported in the literature.

Keywords: Huntington disease/diagnosis, Huntington disease/epidemiology, Huntington disease/genetics, Trinucleotide repeat expansion

DOI: http://dx.doi.org/10.4314/ahs.v15i4.23

Cite as: Boubouche A, Regragui W, Lamghari H, Khaldi K, Birouk N, Lytim S, et al. Clinical and genetic data of Huntington disease in Moroccan patients. Afri Health Sci. 2015;15(4):1232-8. bttp://dx.doi.org/10.4314/abs.v15i4.23
\end{abstract}

\section{Introduction}

Huntington's disease (HD) is a rare neurodegenerative genetic disease, characterized by generalized chorea, dementia, and psychiatric disorders. HD is transmitted as an autosomal dominant trait; children of an affected person have a $50 \%$ risk of inheriting the disease. It

\section{Corresponding author:}

Ahmed Bouhouche,

Department of Neurology and Neurogenetics

Medical School and Pharmacy,

University Mohammed V, Rabat, Morocco

Tel/Fax: 212537775261

E-mail: a.bouhouche@um5s.net.ma is caused by CAG triplet repeat expansions in exon 1 of the Huntingtin gene (IT15) located on chromosome $4 \mathrm{p} 16.3^{1,2}$. Mutant alleles produce a sequence of 36 or more glutamine amino acid in Huntingtin protein which results in a selective neural cell death at the level of the basal ganglia and cerebral cortex ${ }^{3}$. The onset of clinical symptoms varies markedly, usually occurring between the ages of 35 and 55 years, however the disease can manifest at younger age before 14 years or much later after the age of 80 . The age of onset is inversely correlated with CAG-repeat length, which accounts for 50 to $70 \%$ of the variance. Additional genetic and environmental factors seem to modulate the course and the age at onset of the disease ${ }^{4,5,6}$. HD is incurable with death occurring on average 16 years after disease onset ${ }^{7,8}$. 
Worldwide prevalence of HD is 2.71 per 100,000 , but varies greatly with ethnic origin. High prevalence is seen in populations of Western European descent with 5.7 per $100,000^{\circ}$ and much lower prevalence are seen in Japan, China, Finland, and Africa with about one per million people $10,11,12,13$. The highest prevalence has been reported in an isolated population of Venezuela called Lake Maracaibo with up to 700 per 100,000 inhabitants, $30 \%$ of them have been identified as homozygous for the mutant allele ${ }^{14}$. Little is known on people of Arabic origin; the few available studies concerned some countries from the Middle East with a prevalence rate of 3-4 per 100,000 inhabitants ${ }^{15}$.

Huntington's disease was one of the first hereditary neurological diseases for which genetic testing was made possible in 1993. However, up to date there is no publication in the literature reporting patients from North African origin. This present study is the first report of clinical and genetic data characteristics of Huntington patients in Morocco.

\section{Patients and methods}

During the period 2009 to June 2014, 21 HD patients belonging to 17 unrelated families were recruited consecutively from the outpatient clinic of six medical centers in the province of Rabat-Salé (Morocco); the majority of them were seen in the Department of Neurology of Hospital of Spécialités. The 7 related patients belonged to 3 families, a brother and sister, a father and son, and father and 2 children respectively. In the absence of a multidisciplinary counseling committee in Morocco, this study was conducted as part of a research project; and written informed consent was obtained from all subjects. The study was approved by the biomedical research ethics committee of the Medical School of Rabat (CERB). Clinical evaluation was based on a questionnaire including motor symptoms (chorea, bradykinesia, tremor, dysarthria and dysphagia) and cognitive and psychiatric symptoms (memory deficit, loss of motivation, concentration problems, difficulty in managing kitchen utensils, computer, dressing, taking shower, mood problem, insomnia, episode of crying, loss of smiling, ...etc). Genomic DNA was extracted from peripheral blood leukocytes using Isolate II Genomic DNA kit from Bioline. The CAG repeat length was determined as reported by Teo CR et al. ${ }^{16}$. Briefly, 50 ng genomic DNA was amplified using fluorescently labeled primer (HDR-HEX) in a final volume of $25 \mu$ l. One microliter of each PCR product was mixed with $0.5 \mu \mathrm{L}$ of the GeneScan 500HD ROX size standard and $9 \mu \mathrm{L}$ of HiDi Formamide (Applied Biosystems). The mixtures were subjected to capillary electrophoresis on a 310 Genetic Analyzer (Applied Biosystems) for $45 \mathrm{~min}$, and the electrophoretograms were analyzed with Genotyper software version 3.7 (Applied Biosystems). Alleles with CAG repeat size of $36-39$ was defined as reduced penetrant (RP) alleles and $\geq 40$ as fully penetrant (FP) alleles. Allele length less than 36 was considered nonpathogenic ${ }^{17}$. Typical electrophoretograms of normal and affected patients in the study population are shown in Fig. 1. Statistical analysis was conducted using descriptive statistics, the relationship between the repeat length and the age at onset was assessed with the Bravais-Pearson correlation coefficient and statistical significance level was set at $\mathrm{p}<0.05$.

\section{Results}

Twenty one HD patients from 17 unrelated families were diagnosed positive for CAG repeat mutation, of which $8(38 \%)$ were males. There were $4(19 \%)$ sporadic cases and $17(81 \%)$ familial-type HD patients belonging to 13 families. Eleven (65\%) patients inherited the mutation from the father and $6(35 \%)$ from the mother. The mean age at examination was $44.90 \pm$ 10.09 years and the mean age of onset of the disease was $40.71 \pm 8.65$, including a patient with juvenile HD (starting at 5 years) and a patient with late-onset HD (starting at 64). Clinical symptoms were predominantly motor (19/21) mainly chorea, dysarthria and dysphagia. Twelve patients presented with psychiatric and behavioral disorders particularly irritability, apathy and depression. Eleven patients had cognitive disorders essentially of memory impairment, attention deficit and executive dysfunction. 
Figure 1: Electrophoretogram of normal individual and HD patients. The graduate scale in the top indicates the length of the obtained fragment in bp. The size of the CAG repeat is determined from the main band with the highest intensity. $\mathrm{A}, \mathrm{B}$ and $\mathrm{C}$ depict a normal individual, a reduced penetrant $\mathrm{CAG}$ repeat mutation carrier (RP) and a fully penetrant CAG repeat mutation carrier (FP) respectively.

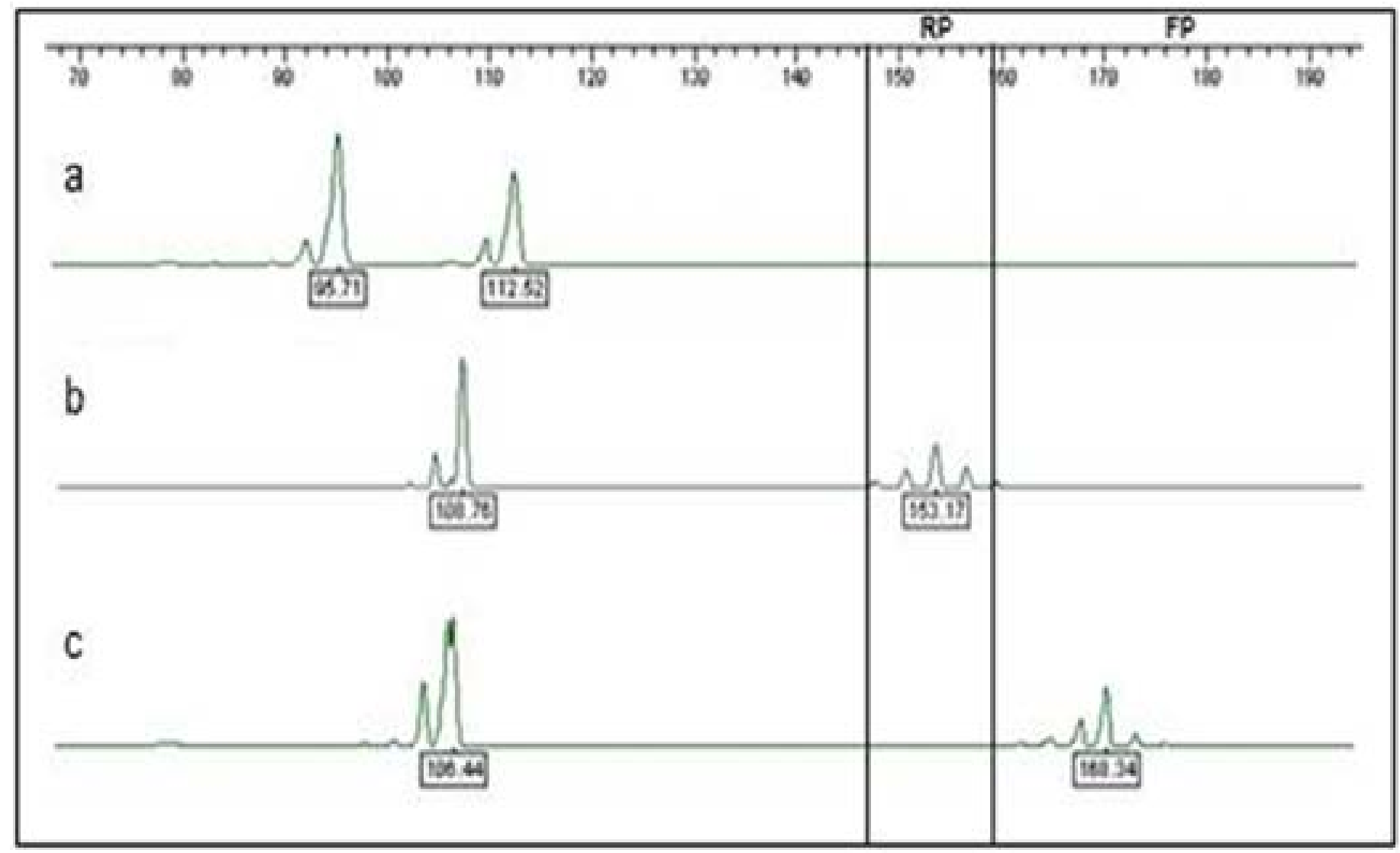

The genetic results showed that the mean number of pathological expanded CAG repeats was $43.8 \pm 4.24$ (range, 38-75). The frequency distribution of the CAG repeat size (Fig. 2) showed that the normal CAG repeats ranged from 9 to 23 repeats with a mode of 16 repeats, whereas the HD CAG repeats ranged from 38 to 75 and the most frequent disease allele was 41 repeats. Fig. 3 shows the relationship between age of onset and the CAG repeat size in affected subjects. The age of HD onset was inversely correlated with the size of the expanded CAG repeats $(\mathrm{r}=-0.754, \mathrm{p}<0.001)$, and the CAG repeat length accounts for up to $56 \%$ of the variance in the age at onset $(\mathrm{R} 2=0.56)$.

Of the $21 \mathrm{HD}$ patients studied, 16 had fully penetrant (FP) and 5, 4 of them were women, had reduced penetrant (RP) CAG repeat alleles (Table 1). The mean CAG repeat size in the FP patient group was $45.5 \pm 4.43$ and was $38.4 \pm 0.48$ in the RP patient group. Although both groups had similar mean disease duration (4 years) their clinical presentation showed some differences. 
Figure 2: Distribution of the normal and expanded CAG repeat alleles in Moroccan HD patients.

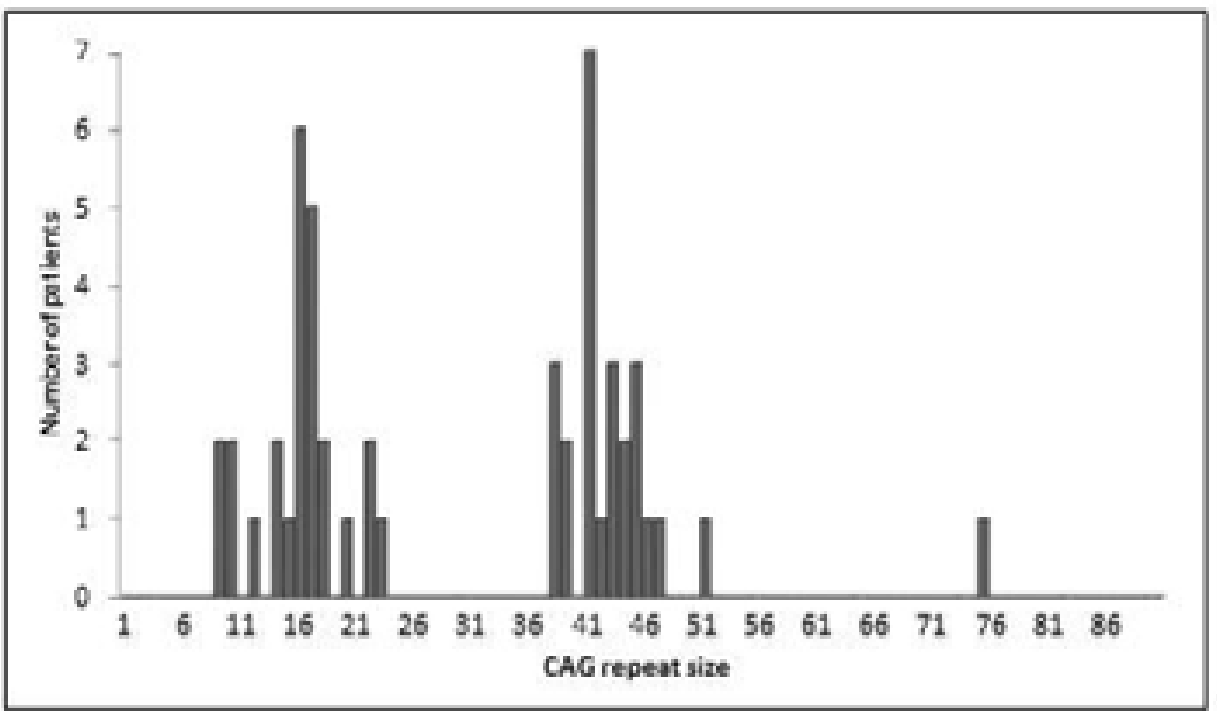

For patients of both groups, the symptom of onset remains largely chorea and motor signs were predom- inant at age of exam. However, psychiatric and behavioral signs were more frequent in FP group than in RP group.

Figure 3: Inverse relationship between the age of onset of the disease and CAG repeat length. Diamonds represent expanded alleles and the diagonal line the regression curve.

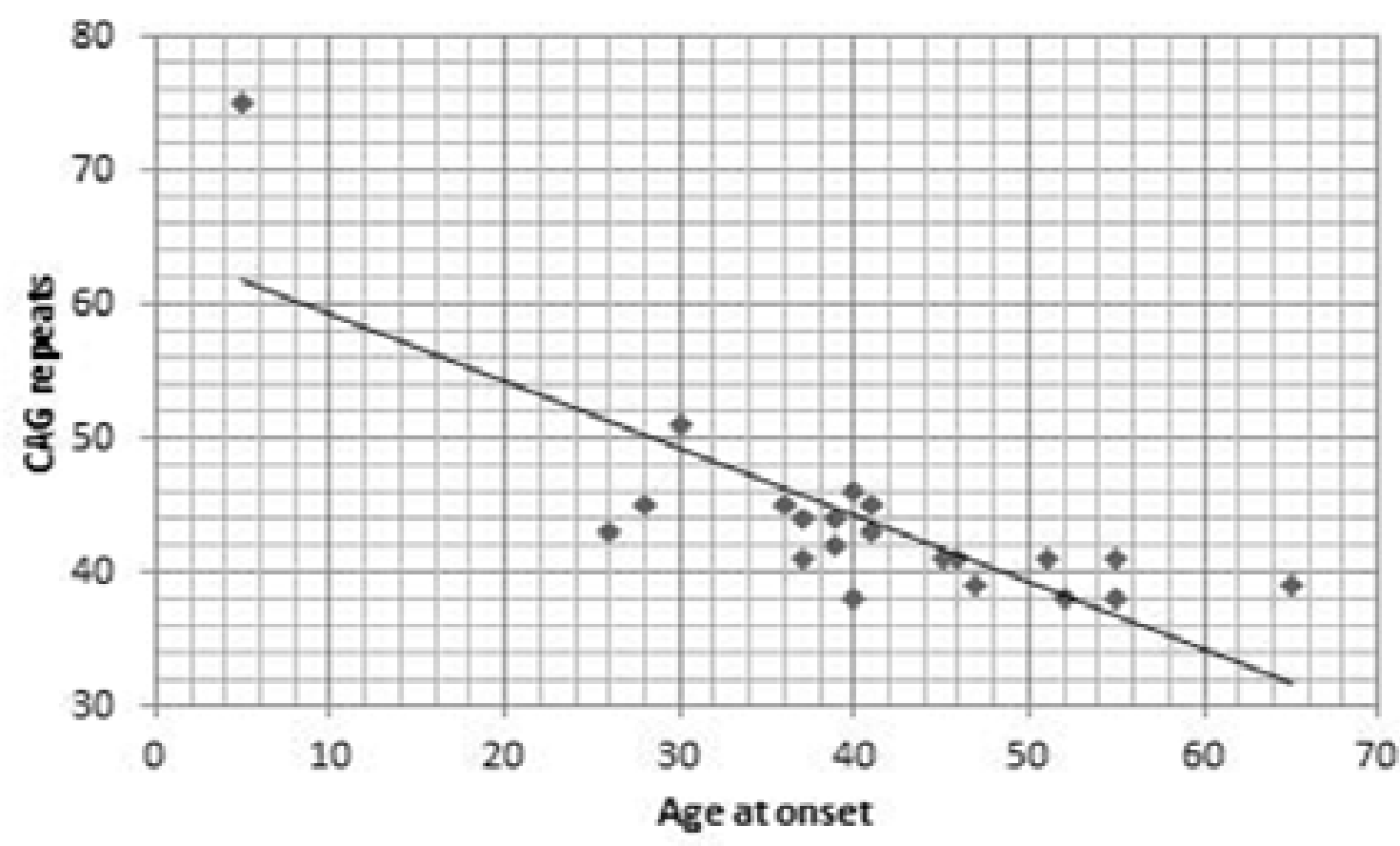


Also, associated signs, more frequent in FP patients, have been seen only in one RP patient who had a disease duration of 10 years including dystonia, akinesia and swallowing disorder (Table 1).

Late onset HD (> 60 years) was seen in one RP patient with CAG repeat size of 39 and who had only choreic movements after one year of disease duration. Juvenile onset HD was seen in 1 FP patient with paternal inheritance, a 7 year old patient with onset in infancy (5 years) who had 75 CAG repeats, and had the typical associated features of rigidity, dyskinesia and dystonia. MRI showed in this patient atrophy of the caudate nucleus and putamen and dilatation of the ventricles ${ }^{18}$.

Table 1: Clinical and genetic data of Moroccan patients with Huntington's disease.

\begin{tabular}{lll}
\hline Patients & FP (N = 16) & RP (N = 5) \\
\hline Mean CAG length & $45.5 \pm 4.43$ & $38.4 \pm 0.48$ \\
Mean age at exam & $41.5 \pm 9.50$ & $55.8 \pm 5.44$ \\
Mean age at onset & $37.2 \pm 7.71$ & $51.8 \pm 6.64$ \\
Mean disease duration & $4.00 \pm 2.70$ & $4.20 \pm 2.32$ \\
Sex (M:F) & $7: 9$ & $1: 4$ \\
$\begin{array}{l}\text { Positive HD family history } \\
\text { Paternal inheritance }\end{array}$ & $13 / 16$ & $4 / 5$ \\
Motor signs & $8 / 13$ & $3 / 4$ \\
$\begin{array}{l}\text { Psychiatric and behavioral } \\
\text { signs }\end{array}$ & $15 / 16$ & $4 / 5$ \\
Cognitive signs & $10 / 16$ & $2 / 5$ \\
Associated signs & $8 / 16$ & \\
Sphincter disturbances & $4 / 16$ & $0 / 5$ \\
Vegetative disturbances & $2 / 16$ & $1 / 5$ \\
Dystonia & $6 / 16$ & $1 / 5$ \\
Ataxia & $2 / 16$ & $0 / 5$ \\
Akinesia & $1 / 16$ & $1 / 5$ \\
Tics & $2 / 16$ & $0 / 5$ \\
\hline
\end{tabular}

FP: Fully penetrant alleles, RP: Reduced penetrant alleles

\section{Discussion}

Over a period of 6 years, $21 \mathrm{HD}$ patients were positive for the IT15 gene CAG repeat recruited from different medical centers in the city of Rabat that collects patients of the Rabat-Salé province with 2 million people. By linear extrapolation, since many HD patients may not be receiving genetic testing particularly those examined by private physicians, the prevalence of $\mathrm{HD}$ in Morocco with 34 million people could be estimated to about 1-4 per 100,000. For a right precise estimate of the prevalence, further epidemiological studies on Huntington disease in Morocco are needed.

HD families of Arab origin have been reported in Oman, Saudi Arabia, Syria, Lebanon, and Egypt. The prevalence rate in this Middle Eastern area was estimat- ed to 3-4 per 100,000 ${ }^{15}$. This is the first study of HD in a Maghreb population composed of Berbers and Arabs. HD was probably introduced in this population by several visitors in earlier time. Indeed, this region originally inhabited by Berbers, saw installation of the Phoenicians (1200 BC) and various invasions including Romans, Vandals and Byzantines and the arrival of the Arabs in the seventh century. The Ottoman Turks arrived in 1550, succeeded the French people who took control of Maghreb territories from 1830.

Four of the twenty one patients $(19 \%)$ with genetically confirmed HD did not have a positive family history. This incidence is similar to that reported in the literature, even in populations with low HD prevalence ${ }^{4,13,19,20}$. These sporadic cases are due to de novo 
mutations resulting generally from expansion of nonpenetrant alleles usually upon paternal transmission ${ }^{21}$. Therefore, genetic diagnosis of patients with negative family history is relevant.

Genetic results showed that the expanded CAG repeat range was between 38 and 75 (mean $=43.8$ ) and that of normal allele range was between 9 and 23. The distribution of the expanded CAG repeat and the significant correlation found for the relationship between the age of onset and the CAG repeat expansion were similar to that for Caucasian population described previously ${ }^{4,13}$. However, the contribution of CAG repeat length in our study accounts only for up to $56 \%$ of the variance in the age at onset. This is lower than reported in some populations such as Venezuela with $85 \%{ }^{5}$ suggesting the existence of other genetic and or environmental factors specific to Moroccan population.

Clinical results showed that HD patients with RP alleles had a moderate clinical presentation consisting of motor and cognitive signs at age of exam. However, the disease could became severe with behavioral and other associated signs such as dystonia and akinesia during the disease evolution as seen in one RP patient with 10 years disease duration. HD patients with FP alleles manifest, in addition to motor and cognitive signs, psychiatric and behavioral signs more frequently than RP patients. The most frequent associated signs in these patients were dystonia and sphincter disturbances. Juvenile HD with very large repeats is very rare and has been seen in our study in only one patient $(1 / 16)$ with 75 CAG repeats who had typical associated features of rigidity and dystonia. His father had 46 CAG repeats with an age at onset of 45 years and a moderate clinical phenotype, indicating an anticipation phenomenon.

\section{Conclusion}

This preliminary study of HD patients in Morocco showed that clinical and genetic data are similar to previous reports. We also demonstrated the relevance of genetic testing of patients with negative family history for HD. The life-long management of patients and families affected by HD is a major problem worldwide. However, since nuclear families are large in Moroccan population and the risk of having several patients in the same family, we highlight in this paper the necessity to create a multidisciplinary council genetic unit in Morocco country for the management of this devastating disease.

\section{Acknowledgements}

The authors would like to thank all the family members for their participation in this study. This work was supported by the Centre National de Recherche Scientifique et Technique (CNRST) of Ministry of High Education and Research, Morocco.

\section{Conflicts of interest}

The authors have no financial conflicts of interest.

\section{References}

1. Gusella JF, Wexler NS, Conneally PM, et al. A polymorphic DNA marker genetically linked to Huntington's disease. Nature 1983; 306(5940): 234-238.

2. Huntington's Disease Collaborative Research Group. A novel gene containing a trinucleotide repeats that is expanded and unstable on Huntington's disease chromosomes. Cell 1993; 72: 971-983.

3. Estrada-Sánchez AM, Rebec GV. Role of cerebral cortex in the neuropathology of Huntington's disease. Front Neural Circuits 2013; 7: 19.

4. Myers RH. Huntington's disease genetics. NeuroRx 2004; 1(2): 255-262.

5. Wexler NS, Lorimer J, Porter J, et al. Venezuelan kindreds reveal that genetic and environmental factors modulate Huntington's disease age of onset. Proc Natl Acad Sci USA 2004; 101(10): 3498-3503.

6. Gusella JF, MacDonald ME. Huntington's disease: the case for genetic modifiers. Genome Medicine 2009; 1(8): 80.

7. Ross CA, Shoulson I. Huntington disease: pathogenesis, biomarkers, and approaches to experimental therapeutics. Parkinsonism Relat Disord. 2009; 15 Suppl 3: S135-138.

8. Trembath MK, Horton ZA, Tippett L, et al. A retrospective study of the impact of lifestyle on age at onset of Huntington disease. Mov Disord 2010; 25(10): 14441450.

9. Pringsheim T, Wiltshire K, Day L, Dykeman J, Steeves $T$, Jette N. The incidence and prevalence of Huntington's disease: a systematic review and meta-analysis. Mov Disord 2012; 27(9): 1083-1091.

10. Chang CM, Yu YL, Fong KY, et al. Huntington's disease in Hong Kong Chinese: epidemiology and clinical picture. Clin Exp Neurol 1994; 31: 43-51.

11. Nakashima K, Watanabe Y, Kusumi M, et al. Epidemiological and genetic studies of Huntington's disease in the San-in area of Japan. Neuroepidemiology 1996; 15(3): 126-131. 
12. Hayden MR, MacGregor JM, Beighton PH. The prevalence of Huntington's chorea in South Africa. $S$ Afr Med J 1980; 58(5): 193-196.

13. Agostinho LA, Dos Santos SR, Alvarenga RM, Paiva CL. A systematic review of the intergenerational aspects and the diverse genetic profiles of Huntington's disease. Genet Mol Res 2013; 12(2): 1974-1981.

14. Avila-Giron R. Medical and Social Aspects of Huntington's chorea in the state of Zulia, Venezuela. Advances in Neurology 1973; 1: 261-266.

15. Scrimgeour EM. Huntington disease (chorea) in the Middle East. Sultan Qaboos Univ Med J 2009; 9(1): 16-23.

16. Teo CR, Wang W, Yang Law H, Lee CG, Chong SS. Single-step scalable-throughput molecular screening for Huntington disease. Clin Chem 2008; 54(6): 964-972.
17. Potter NT, Spector EB, Prior TW. Technical standards and guidelines for Huntington disease testing. Genet Med 2004; 6(1): 61-65.

18. Nejjari I, Rhouda H, Bouhouche A, Y. Kriouile Y. Juvenile Huntington disease: A case study. Archives de Pédiatrie 2013; 20: 1161-1163.

19. Shin CW, Choi YJ, Kim M, Jeon BS. Preliminary analysis of Huntington's disease in South Korea.J Huntingtons Dis 2013; 2(1): 83-87.

20. Sumathipala DS, Jayasekara RW, Dissanayake VH. Clinical and genetic features of Huntington disease in Sri Lanka. BMC Neurol 2013; 13: 191.

21. Aziz NA, van Belzen MJ, Coops ID, Belfroid RD, Roos RA. Parent-of-origin differences of mutant HTT CAG repeat instability in Huntington's disease. Eur J Med Genet 2011 ; 54(4):e413-418. 